\title{
Shape Extraction Methods for Fruits: Technical Review
}

\author{
Divya Kakadiya \\ Students of Integrated \\ M.Sc.(IT) \\ At Department of Computer \\ Science and Technology, UTU \\ Bardoli, Gujarat. \\ Riya Shah \\ Students of Integrated \\ M.Sc.(IT) \\ At Department of Computer \\ Science and Technology, UTU \\ Bardoli, Gujarat.
}

\author{
Nirali Shah \\ Students of Integrated \\ M.Sc.(IT) \\ At Department of Computer \\ Science and Technology, UTU \\ Bardoli, Gujarat. \\ Chandres Kachariya \\ Students of Integrated \\ M.Sc.(IT) \\ At Department of Computer \\ Science and Technology, UTU \\ Bardoli, Gujarat.
}

\author{
Munika Patel \\ Students of Integrated \\ M.Sc.(IT) \\ At Department of Computer \\ Science and Technology, UTU \\ Bardoli, Gujarat. \\ Krunal Sukhwani \\ Teaching Assistant \\ At Department of Computer \\ Science and Technology, UTU \\ Bardoli, Gujarat.
}

\begin{abstract}
Fruit categorizations in agriculture industry have upgraded from traditional grading to automatic grading over the past 25 years. To identify object residing in image, the image has to be described or represented by certain features. Shape is an important visual feature of an image. Shape extraction has attracted much attention lately. Many shape representation and description techniques are discuss in this review paper. Shape extraction techniques play an important role in systems for object recognition, matching, extracting, and analysis. It also presents comparison between various techniques.
\end{abstract}

\section{KEYWORDS}

Shape Extraction, Object recognition, Shape, Object Extraction, shape representation

\section{INTRODUCTION}

Many companies are moving to automated grading in many crops such as grading on peaches and oranges [4]. Modern time is an era of technology in which to believe in vision based intelligence. The use of the digital images has been tremendous in day-to-day life. Image processing performs major role in science and technology. Digital image processing technology has become an efficient and cost effective technology, so shape of the objects has been explores using several techniques with concrete results. On the basis of digital vision, it requires to identify to shape of object residing in image. The production of high-quality food depends not only on an appropriate processing technology but also on the selection of proper raw materials. This experience, well known among food manufactures, can be exemplified in the case of mango processing [10].

Computer vision provides different solutions to reduce the human effort in the field of agriculture. Researchers have focused on several topics as finding weeds, searching for fruits, monitoring the health status of trees, automatic classification of fruits, determining fruit ripeness, detecting diseases in trees and fruit [3].

Shape Extraction is a process of identifying and extracting shape of object from digital image. Shape extraction is typically used to locate objects and boundaries (lines, curves, etc.) of images [18]. For that first step is require to perform segmentation. Image segmentation is the process of dividing a digital image into multiple segments (sets of pixels). The aim of segmentation is to simplify and change the representation of an image into something more meaningful and easier to analyse. Segmentation algorithms are based on one of two basic properties of intensity values discontinuity and similarity. First category is to partition an image based on abrupt changes in intensity, such as edges in an image. Second category is based on partitioning an image into regions that are similar according to predefined criteria [9]. Several steps are needed in order to detect the object through the colour processing [28-30]. By referring to the Figure 1, the images are passed by the pre-processing block. After that, it will directly go through the RGB arrangement where it will readjust the lighter and darker the object pixels colour and background. Next, colour processing take place and the elimination of the unrelated colour would be proceed and left the colour of the mango. Then the current pixel RGB value is compared with the default RGB value of object. If detected colour is not related then change the current pixel RGB value to 0 which is in black colour.

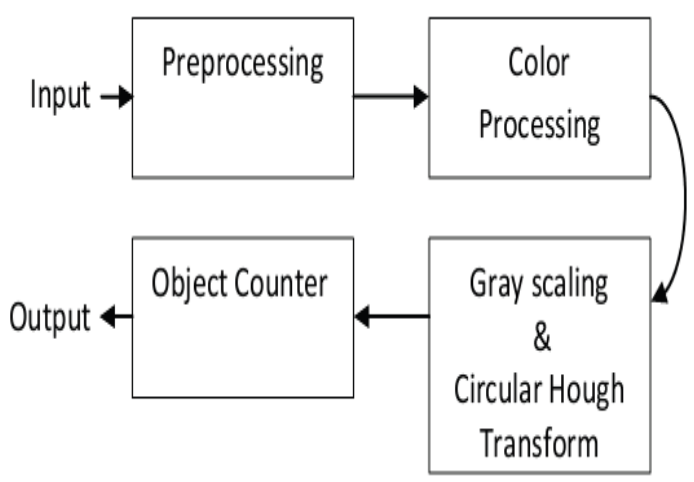

Figure 1: Fruits recognition Block Diagram [15]

The process of object retrieval has become an active research topic because of its application in many fields like Biometric systems, Image Search Engines, Digital Libraries, Medical Records etc., There are several applications for shape extraction like Auto-farming systems, automatic fruit/vegetable harvesting systems, automatic fertilization systems, automatic irrigation-spraying systems, the automated strawberry grading system, Mango grading system [4][20][21]. In addition computer vision technology can easily be adapted to other applications such as on tree yield monitoring, crop health status monitoring, disease detection, and other operations which require vision sensor output [6]. Practically, extracting fruits from images requires initial processes in order to determine the initial objects and then 
verify whether the objects represent fruit or do not. These processes isolate areas of fruit from the background (background contains sky, leaves, branches, ground, noise, etc.), whether by converting colour image to grey or binary colour image by applying a certain threshold, by using certain morphological conversion such as watershed or by applying some of colour segmentation methods such as in the proposed methods implemented to extract tomato, apple and eggplant [3].

Here, Section 2 describes literature review for various shape analysis and representation techniques, algorithms and different segmentation methods, used in various automatic grading systems. Further section presents classification of shape representation technique namely, Contour-based and Region-based. And it also gives brief idea of few techniques.

\section{LITERATURE REVIEW}

Fruits are easy to handle and to help satisfy consumer preferences. All the fruits have their length, maximum width and maximum thickness using mechanical vernier caliper. Shape of mango 'Chock Anan'(type of mango) fruit were measured with length of mango, maximum width of mango and maximum thickness of mango. An equation to calculate the shape of the mangoes based on three geometric dimensions, which then showed a high level of explanatory power of $\mathrm{R} 2=0.97$. The root mean standard error of 12.22 was determined, and the mean relative deviation found was 0.05 . Correlation $(\mathrm{R} 2=0.96)$ was found between measured mass and calculated mass [2]. Shape is an attribute that affects the performance of many fruit quality sensors. Shape comparison and classification can be defined as the systematic arrangement of shapes within a database, based on some similarity criteria. These criteria are gathered in a similarity metric [1]. Similarity metrics can be divided into those based on feature matching and those based on dense matching. Dense matching methods try to transform one shape into another based on some energy optimization scheme, and they are computationally expensive [28].

In [3], they have proposed method that is based on preprocessing operators. It convert into Gary scale image, second find edges, after this calculating distances to edges and last convert into binary colour image. It extracts hypothetic places of mango fruit using Randomized Hough Transform method which use Back propagation Neural Network

In [4] it, Images were taken at different time and duration simultaneously in different condition for batter result. It proposes method to detect orange on tree. There are steps like local analysis and shape analysis. In local analysis applied method, first step is segmentation which extracts the orange fruit from brown branches, green leaves, and may be sky of image. It generates colour histogram of the orange fruit. In Shape analysis, colour image is converting into black and white image (binary image) after segmented by the local analysis.

There are different types of Image segmentation methods can be categorized that region based techniques, clustering technique, Split and Merge Technique, Thresholding, edge based techniques [23]. In this Image segmentation classified into two types: Local segmentation and global segmentation. Pattern recognition and image analysis are basic steps for image segmentation. Artificial intelligence is one of the methods of image processing which include fuzzy logic. Segmentation based on pixel values is the easiest approach [22]. Threshold method as existing technology and it is difficult to select threshold. It proposes method where it automatically gets the threshold using histogram analysis [8]. According [23] to the different types of research methods, it can be divided into three levels: Image processing, image analysis and image understanding.

This [24] paper describe image processing \& analysis techniques for finding weight of mango. Size of mango is estimate by weight. Relationship between pixel of mango and weight was analysed with the help of statistical method.

There are two possible approaches for 2D shape description within the context of feature matching methods. One relies on the definition of a set of landmark points, while the other works with the whole contour or region, respectively, of the shape [28].

Many image retrieval techniques are based on textual disputation of images. Content based image retrieval uses the visual contents of an image such as colour, shape, texture, and spatial layout to represent and index the image. The Region Based Image Retrieval (RBIR) system uses the Discrete Wavelet Transform (DWT) and a Watershed. The Region Based Image Retrieval (RBIR) system uses the Discrete Wavelet Transform (DWT) and a Watershed [19]

This [17] propose algorithm for detecting and sorting mango with help of captured image. Features of mango were collected to identify type of mango. Mangoes are classified with the help of its physical features. Structure of mangoes can help in classification process. Object's pixels and defective area is classified with the help of threshold technique. This algorithm describes green and brown pixels. Green pixels are considered as good area and Brown pixels are considered as defective area. Threshold segmentation techniques classify in three classes: First steps based on the local properties of neighbouring pixels. Second global techniques segment an image on the basis of information obtain globally. Third Split and merge techniques use both the observation of uniformity and geometrical proximity in order to get good segmentation results [9].

In Hough transform, the pixels points are shackled by determining first if they lie on the curve of specified shape. In theory, it can be used to find features of any shape in an image. In practice it is only generally used for finding straight lines or circles. The computational complexity of the method is increased rapidly with more complex shapes. It is a kind of Brute-Force method and very complex in computation. It has two main drawbacks: large memory requirement and slowness [12].

\section{METHODS AND ALGORITHMS}

There are several methods and algorithms for shape extraction namely, Circular Hough Transform (CHT), Discrete Wavelet Transform (DWT), Fourier Transform of Boundary, Region Growing Algorithm, Chain Code, Scale Space, Clustering .

Generally, they are classified into two approaches namely contour-based and region-based. Under each class, the methods can be divided into structural and global methods.

Contour-based approaches are more popular than region-based approaches in theory. This is because human beings are thought to discriminate shapes mainly by their contour features. Another reason is because in many of the shape applications, the shape contour is the only interest, whilst the shape interior content is not important. 


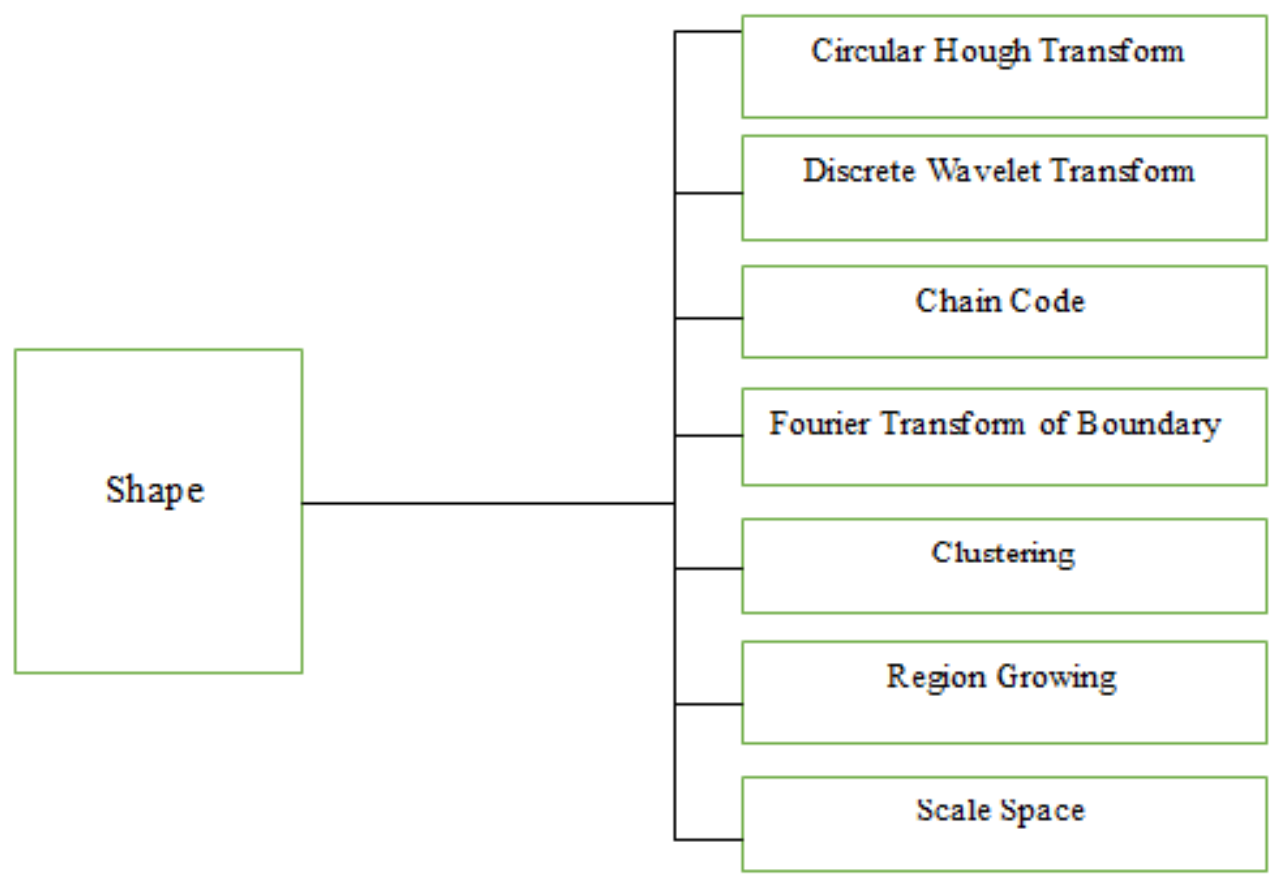

Figure 2: Techniques of shape extraction

\section{Circular Hough Transform (CHT):}

In [12][15][16] CHT, it performs simple mathematical calculation such as circular formula. In here, it detect the location of "1" from the binary image and from there, it perform the calculation and determine the circular objects. To detect it, the radius of the object must be given in order to detect the required round objects size. It detects $60 \%$ of it. In this step, some unrelated object are detected due to it has the similar circular area because of the CHT are detect the object base on the edge of each object.

The equation of the a circle is,

$\mathrm{r}^{2}=(\mathrm{x}-\mathrm{a})^{2}+(\mathrm{y}-\mathrm{b})^{2}$

Here $\mathrm{a}$ and $\mathrm{b}$ represent the coordinates for the centred, and $\mathrm{r}$ is the radius of the circle.

The parametric representation of this circle is

$\mathrm{x}=\mathrm{a}+\mathrm{r}^{*} \cos (\theta)$

$\mathrm{y}=\mathrm{b}+\mathrm{r} * \sin (\theta)$

\section{Discrete Wavelet Transform (DWT):}

It is multi-resolution analysis and it decomposes images into wavelet coefficients and scaling functions. In it signal energy concentrates to specific wavelet coefficients. This characteristic is useful for compressing images [31]. Wavelets convert the image into a series of wavelets that can be stored more efficiently than pixel blocks. Wavelets have rough edges; they are able to render pictures better by eliminating the noise pixels. Image consists of pixels that are arranged in two dimensional matrixes, each pixel represents the digital equivalent of image intensity. In spatial domain adjacent pixel values are highly correlated and hence redundant. In order to compress images, these redundancies existing among pixels needs to be eliminated. DWT processor transforms the spatial domain pixels into frequency domain information that are represented in multiple sub-bands, representing different time scale and frequency points. It is classified as Haar Transform and Daubechies wavelet [27].

\section{Fourier Transform of Boundary:}

The shape description methods under this category use the Fourier Transform of the one dimension boundary representation and Region-based Fourier descriptor to characterize the shape.

One-dimensional Fourier descriptors:

Fourier descriptor (FD) is obtained by applying Fourier transform on a shape signature that is a one-dimensional function which is derived from shape boundary coordinates. The normalized Fourier transformed coefficients are called the Fourier descriptor of the shape. FD derived from different signatures has significant different performance on shape retrieval [33-35].

\section{Region-based Fourier descriptor:}

The region-based FD is referred to as generic FD, which can be used for general applications. Basically, GFD is derived by applying a modified polar Fourier transform (MPFT) on shape image [57, 12]. In order to apply MPFT, the polar shape image is treated as a normal rectangular image. The steps are

1. The approximated normalized image is rotated counter clockwise by an angular step sufficiently small.

2. The pixel values along positive $\mathrm{x}$-direction starting from the image centre are copied and pasted into a new matrix as row elements.

3 . The steps 1 and 2 are repeated until the image is rotated by $360^{\circ}[33-35]$

\section{Region Growing Algorithm:}

In [11], a region in an image can be defined by its border (edge) or its interior. 
Region Growing: The concept of region growing algorithm is to check the neighboring pixels of the initial seed points, then determine whether those neighboring pixels are added to the seed points or not.

\section{Select the seed points.}

2. If the neighbor pixels of the initial seed points satisfy the criteria such as intensity, gray level texture, and color, they will be grown.

3. Use the criteria of the same pixel value, and then check the neighboring pixels of the initial seed points. If their pixel values match with seed points, they can be added to the seed points. It stops until there is no change in two successive iterations.

\section{Scale Space:}

This group contains methods that rely on the scale-space representation. In scale space theory a curve is embedded into a continuous family $\left\{\mathrm{T}_{a}: a \geq 0\right\}$ of gradually simplified versions. Scale-space filtering approach provides a useful representation for representing significant object features. The representation was created by tracking the position of infection points in signals filtered by low-pass Gaussian filters of variable widths. The infection points that remained present in the representation were expected to be "significant" object characteristics. The concept of multi-scale filtering is also present in mathematical morphology. Thus due to different

\section{METHOD COMPARISON}

\begin{tabular}{|c|c|c|}
\hline & Advantages & Disadvantages \\
\hline Region-Growing Technique & $\begin{array}{l}\text { 1) Work best when the region uniformity } \\
\text { measurement is easy to define. }\end{array}$ & $\begin{array}{l}\text { 1) It is quite expensive both in } \\
\text { computational time and memory. } \\
\text { 2) Region growing is dependence on the } \\
\text { selection of seed. }\end{array}$ \\
\hline $\begin{array}{l}\text { Circular Hough } \\
\text { (CHT): }\end{array}$ & $\begin{array}{l}\text { 1) Easy implementation. } \\
\text { 2) Handles missing and occluded data very } \\
\text { gracefully. }\end{array}$ & $\begin{array}{l}\text { 1) Computationally is complex for objects } \\
\text { with many parameters. } \\
\text { 2) The length and the position of a line } \\
\text { segment cannot be determined. } \\
\text { 3) Co-linear line segments cannot be } \\
\text { separated. }\end{array}$ \\
\hline Discrete Wavelet Transform & $\begin{array}{l}\text { 1) Wavelets are well localized in both time } \\
\text { and frequency domain whereas the standard } \\
\text { Fourier transform is only localized in } \\
\text { frequency domain. }\end{array}$ & $\begin{array}{l}\text { 1) The disadvantage is loss of generality. } \\
\text { 2) Wavelets have not been used widely in } \\
\text { image processing due to the difficulty in } \\
\text { designing complex filters which satisfy a } \\
\text { perfect reconstruction property. }\end{array}$ \\
\hline $\begin{array}{lll}\text { Fourier } & \text { Transform } & \text { of } \\
\text { Boundary: } & & \end{array}$ & $\begin{array}{l}\text { 1) The major advantage of this method is that } \\
\text { it is easy to implement and based on a well- } \\
\text { developed theory of Fourier analysis. }\end{array}$ & $\begin{array}{l}\text { 1) Fourier transform does not provide local } \\
\text { shape information. After the Fourier } \\
\text { transform, local shape information is } \\
\text { distributed to all coefficients and not } \\
\text { localized in the frequency domain. }\end{array}$ \\
\hline Clustering & 1) less computational complexity & $\begin{array}{l}\text { 1) A major disadvantages of cluster } \\
\text { sampling is that this method tends to } \\
\text { produce less representative samples } \\
\text { compare to other probability sampling } \\
\text { designs, particularly when the cluster } \\
\text { contain large numbers of units within them } \\
\text { and only a few are needed to meet desired } \\
\text { sample size }\end{array}$ \\
\hline
\end{tabular}

scales, it is possible to separate small details from relevant shape properties. [33][34].

\section{Chain code:}

Chain code is used for identifying different shapes as line drawings, planar curves, or contours. Chain code uses an object by a sequence of unit size line segments with a given orientation [36]. A chain is an ordered sequence of links written in the form $\mathrm{A}=\mathrm{a} 1 \mathrm{a} 2 \mathrm{a} 3 \ldots . . . \mathrm{an}$. In addition to these basic operations it is possible to compute the inverse and length of a chain, the integral of a function specified by its chain code, the first and second moments about $\mathrm{x}$ and $\mathrm{y}$ axes, and the distance between two points connected by a chain. The above operations illustrate the edibility and versatility of the chain code for algorithm realization. The shape boundary is represented in a hierarchical way so that at the highest level a lower number of polygon vertices is used, while at the lowest level the finest polygonal approximation is utilized. Chain code can be viewed as a connected sequence of straight-line segments with specified lengths and directions [29].

\section{Clustering:}

Clustering is mechanism for grouping set of pixels in such a way that pixels in the same group are more similar to each other than to those in other groups. Popular notions of clusters include groups with small variance among the pixels value. 


\begin{tabular}{|l|l|l|}
\hline Chain Code & $\begin{array}{l}\text { 1) The advantage is reduction in storage } \\
\text { volume }\end{array}$ & $\begin{array}{l}\text { 1) The disadvantage is loss of generality. } \\
\text { 2) The basic chain code is very sensitive to } \\
\text { noise \& it is not rotationally invariant }\end{array}$ \\
\hline Scale Space: & $\begin{array}{l}\text { 1) The great advantages are the high } \\
\text { robustness to noise and the great coherence } \\
\text { with human perception. }\end{array}$ & $\begin{array}{l}\text { 1) Computational complexity is average in } \\
\text { scales-space method. }\end{array}$ \\
\hline
\end{tabular}

\section{CONCLUSION}

In this review paper, many techniques for extraction of shape features have been shortly described and compared. The approaches of shape-based feature extraction and representation were classified into contour-based and regionbased. Extracting a shape feature in accordance with human perception is not an easy task. Due to the fact that human vision and perception are an extraordinary complicated system, it is a utopia to hope that the machine vision has super excellent performance with small complexity. There exists no general feature which would work best for every kind of images.

Future Work: we intend to count number of mangoes from digital image.

\section{REFERENCES}

[1] G.P. Moreda, M.A. Muñoz, M. Ruiz-Altisent, A. Perdigones, "Shape determination of horticultural produce using two-dimensional computer vision - A review", Journal of Food Engineering 1082012.

[2] Wolfram Spreer, Joachim Müller, "Estimating the mass of mango fruit from its geometric dimensions by optical measurement", Computers and Electronics in Agriculture 75,2011

[3] Kutiba Nanaa, Mohamed Rizon, Mohd Nordin Abd Rahman, Yahaya Ibrahim and Azim Zaliha Abd Aziz ,"Detecting Mango Fruits by using Randomized Hough Transform and Backpropagation Neural Network", 18th International Conference on Information Visualisation ,2014.

[4] Yüksel Çakır, Mürvet Kırc1, Ece Olcay Güneş , "Detection of Oranges In Outdoor Conditions", Department of Electronics and Communication Engineering.

[5] A.B. Payne , K.B. Walsh b, P.P. Subedi, D. Jarvis "Estimation of mango crop yield using image analysis Segmentation Method", Computers and Electronics in Agriculture 912013.

[6] Tajul Rosli B. Razak, Mahmod B. Othman, Mohd Nazari bin Abu Bakar, Khairul Adilah Ahmad, Ab Razak Mansor , "Mango Grading By Using Fuzzy Image Analysis", International Conference on Agricultural, Environment and Biological Sciences (ICAEBS'2012) May, 2012.

[7] Ankur M Vyas, Bijal Talati, Sapan Naik "Quality Inspection and Classification of Mangoes using Color and Size Features", International Journal of Computer Applications .
[8] Pritee Gupta ,Vandana Malik and Mallika Gandhi , “ Implementation of Multilevel Threshold Method for Digital Images Used In Medical Image Processing ," International Journal of Advanced Research in Computer Science and Software Engineering, February 2012

[9] Salem Saleh Al-amri, N.V. Kalyankar and Khamitkar S.D, "Image Segmentation by Using Thershold Techniques", JOURNAL OF COMPUTING, MAY 2010.

[10] http://link.springer.com/chapter/10.1007\%2F978-3-54071220-6_13\#page-2 date:03/01/2015

[11] C.J. Kuo,Chia-Hao Tsai,"Fast Image Segmentation and Boundary Description Techniques", Graduate Institute of Communication Engineering,M.S. thesis, National Taiwan Univ., 2010.

[12] Mamta.Juneja and Parvinder S. Sandhu, "A New Approach for Feature Extraction of an Image", 3rd International Conference on Intelligent Computational Systems (ICICS'2013), 2013.

[13] Guangyu Liu,Hongyu Bian ,Hong Shi ," Sonar Image Segmentation based on an Improved Level Set Method", 2012 International Conference on Medical Physics and Biomedical Engineering.

[14] G.P. Moreda, J. Ortiz-Cañavate, F.J. García-Ramos, M. Ruiz-Altisent, "Non-destructive technologies for fruit and vegetable size determination - a review", Journal of Food Engineering 92, 2009.

[15] R. Hussin, M. Rizon Juhari, Ng Wei Kang, R.C.Ismail, A.Kamarudin, "Digital Image Processing Techniques for Object Detection From Complex Background Image", International Symposium on Robotics and Intelligent Sensors 2012.

[16] S. Bindu, S. Prudhvi, G. Hemalatha, Mr. N. Raja Sekhar, Mr V. Nanchariah5 , " Object Detection from Complex Background Image Using Circular Hough Transform, Int. Journal of Engineering Research and Applications , April 2014.

[17] Tomas U. Ganiron Jr., " Size Properties of Mangoes using Image Analysis", International Journal of BioScience and Bio-Technology, 2014.

[18] Krishna Kant Singh, Akansha Singh, " A Study Of Image Segmentation Algorithms For Different Types Of Images", International Journal of Computer Science , September 2010

[19] Niket Amoda, Ramesh K Kulkarni, "Image Segmentation and Detection using Watershed Transform and Region Based Image Retrieval, International Journal of Emerging Trends \& Technology in Computer Science (IJETTCS). 
[20] Xu Liming and Zhao Yanchao, "Automated strawberry grading system based on image processing," Computers and Electronics in Agriculture, April 2010.

[21] Tajul Rosli Bin Razak, Mahmod Bin Othman(DR), Mohd Nazari Bin Abu Bakar(DR), Khairul Adilah BT Ahmad, and AB.Razak Bin Mansor, "Mango Grading By Using Fuzzy Image Analysis," in In proceedings of International Conference on Agricultural, Environment and Biological Sciences, 2012.

[22] H.P. Narkhede, "Review of Image Segmentation Techniques", International Journal of Science and Modern Engineering (IJISME), July 2013.

[23] Split and Merge Technique, "COLOUR IMAGE SEGMENTATION TECHNIQUES AND ISSUES: AN APPROACH", International Journal of Scientific \& Technology, May 2012

[24] C.C. Teoh and A.R. Mohd Syaifudin, "Image processing and analysis techniques for estimating weight of Chokanan mangoes", J. Trop. Agric. and Fd. Sc., 2007.

[25] Jagadeesh Devdas Pujari1, Rajesh Yakkundimath2 and Abdulmunaf Syedhusain Byadgi, "Grading and Classification of Anthracnose Fungal Disease of Fruits based on Statistical Texture Features", International Journal of Advanced Science and Technology, March, 2013

[26] Ashraf A. Aly1, Safaai Bin Deris2, Nazar Zaki3," RESEARCH REVIEW FOR DIGITAL IMAGE SEGMENTATION TECHNIQUES", International Journal of Computer Science \& Information Technology (IJCSIT), Oct 2011.

[27] Dipalee Gupta1, Siddhartha Choubey, "Discrete Wavelet Transform for Image Processing", International Journal of Emerging Technology and Advanced Engineering, March 201.5

[28] Ding, W., Nesumi, H., Takano, Y., Ukai, Y., "Quantitative evaluation of the threedimensional fruit shape and size of citrus species based on spherical harmonic descriptors",2000.
[29] D’Orizio, T., C.Guaragnella, M. Leo and A. Distante, ”A new algorithm for ball recognition using circle Hough Transform and neural classifier", Pattern Recognition,2004.

[30] Yazid, H., M. Rizon, P Saad, A.Y.M. Shakaff, S. Yaacob, A.R.M. Saadand M. Sugisaka, 2005. "An approach of coconuts detection using edge information". Proc. of Intl. Advanced Technology Cong.

[31] Mohamed Rizon, H. Y., Puteh Saad, Ali Yeon Md Shakaff, Abdul Rahman Saad, "Object Detection using Circular hough Transform”, 2005.

[32] Ms.Yamini S.Bute, Prof. R.W. Jasutkar, 2012, "Implementation of Discrete Wavelet Transform Processor For Image Compression", International Journal of Computer Science and Network (IJCSN), Vol. 1.

[33] Suchitra Khoje, Shrikant Bodhe, "Performance Comparison of Fourier Transform and Its Derivatives as Shape Descriptors for Mango Grading", International Journal of Computer Applications, September 2012.

[34] Seven Loncaric, "A survey of shape analysis techniques", Department of lectronic Systems and information processing, Faculty of Electrical Engineering and Computing, University of Zagerb.

[35] Dengsheng Zhang, Guojun Lu, "Review of shape representation andd escription techniques",Gippsland School of Computing and Info. Tech., Monash University, July, 2003

[36] Yang Mingqiang, Kpalma Kidiyo, and Ronsin Joseph, "A Survey of Shape Feature Extraction Techniques", Shandong University.

[37] D. Zhang and G. Lu, "Review of shape representation and description techniques," PatternRecognition, 2004

[38] Musoko Victor, Proch'Azka Ale's, “Complex Wavelet Transform in signal and Image analysis", Institute of Chemical Technology, Department of Computing and Control Engineering. 